\title{
Design and Development of MSA agent for Rectangular and U Slotted MSA
}

\author{
Aruna Rani \\ G.B. Pant Engineering College, Pauri \\ Uttarakhand, India
}

\author{
A.K. Gautam \\ G.B. Pant Engineering College, \\ Pauri, Uttarakhand, India
}

\begin{abstract}
Recently, research interest has increased in the design, development, and deployment of microstrip antenna agent systems for high-level inference and surveillance in a wireless sensor network (WSN). The proposed Antenna agent systems employ migrating codes to facilitate flexible application retasking, local processing, and calculating parameters. This provides extra flexibility, as well as new capabilities to MSA. It employs the genetic Algorithm to generate various results. This provides extra flexibility as well as new capability to design Rectangular and U slotted Microstrip Antenna.The development of Rectangular \& U slit loaded antenna into Algorithm design based on parameter analysis and the coding for the architecture of rectangular and U slot loaded MSA agent. The taxonomy covers low level to high level design issue and facilitate the variation in design parameters like $\mathrm{h}, \mathrm{fr}, \boldsymbol{\varepsilon} \mathrm{r}$, flow, fhigh, $\mathrm{R}$ according to the requirement of the proposed agent.
\end{abstract}

IndexTerms-- Microstrip antenna, communication, dielectric constant, frequency, bandwidth, java program, algorithm, Optimization program, genetic algorithm, SHF, U slot.

\section{INTRODUCTION}

Micro strip patch antennas (MPAs) have attracted widespread applications due to their small size, light weight, low profile and low cost as well as to the fact that they are simple to manufacture, suited to planar and non planar surfaces, mechanically robust, easily integrated with circuits, allow multi frequency operation to be achieved [8].The Software for accurately design MSA[1], within minimum time and cost with added features, is in booming demand today in the field of wireless communication[2]. The MSA agent is special kind of software or a computer program that helps to design rectangular and U slot loaded MSA[3] at any resonating frequency[4] within its design limitations. The use of Genetic algorithm[5] made it a potential program. The parametric study can also be carried out by the proposed program. It performs the task autonomously and intelligently in response to calculate the bandwidth[6] and gain[7] of rectangular and U slotted MSA. The parameter passing is on hit and trial basis the GA searches the various results to design the MSA.

Though many software are present today like EM simulator software, IE3D to design micro strip antennas but they are very much time consuming, complicated and are less user friendly. The MSA agent will add a new chapter in this world for designing rectangular and $U$ slotted micro strip antenna. Which is capable to generate various design parameters accurately within a second. It is a Graphical User Interface based user friendly software. It is very simple to use for specific proposed shape antenna. The software is based Genetic algorithm and development on java platform is shown in figure 1. Genetic Algorithm is a class of search techniques that use the mechanisms of natural selection and genetics to conduct a global search of the solution and this method can handle the permittivity, shape and the dimensions for $\mathrm{U}$ slot and the rectangular micro strip antenna. According to the probability of mutation, the chromosome are chosen at random and any one bit chosen at random is flipped from " 0 " to " 1 " or vice versa. After mutation has taken place, the fitness is evaluated. Then the old generation is replaced completely or partially. This process is repeated. After a while all the chromosome and associated fitness become same except for those that are mutated. At this point the genetic algorithm has to be stopped The Genetic Algorithm program, for the optimization of micro strip antenna using this program. The bandwidth variation is analyzed by changing the substrate material at the frequency range $4 \mathrm{GHz}$ to 6 $\mathrm{GHz}$ by introducing the $\mathrm{U}$ slot in the patch. The results are simulated with java optimization program . Stack configuration with 2 patches, driven and parasitic, and the use of the various substrate loading technique increases the bandwidth of the antenna ranging from $48 \%-63 \%$.

The proposed micro strip antenna design agent has a number of attractive features including:

- It execute on any platform, the Java Run Environment made it easier to access for the user.

- It analyze a micro strip antenna structure using different dielectric layers.

- Simultaneously two types of antennas can be design.

- Easy to use which will promote student interest and learning in the field of antenna design.

- Slot coupled antennas can be modeled and design.

- It can be used for parametric study of micro strip antenna.

- It has controlled integration accuracy.

\section{THE GENETIC ALGORITHM}

Genetic Algorithms (GAs) : are adaptive heuristic search algorithm premised on the evolutionary ideas of natural selection and genetic. The basic concept of genetic algorithm is designed to simulate the process in natural system necessary for evolution, specifically those that follow the principles laid down by Charles Darwin of survival of the fittest. As such they represent an intelligent exploitation of a random search within a defined search space to solve a problem.

The Genetic Algorithm has proposed by John Holland in the 60s, then genetic algorithms has been widely studied, experimented and applied in many fields in engineering worlds. Not only does GAs provide an alternative methods to solving problem, it consistently outperforms other traditional methods in most of the problems link. Many of the real world problems involved finding 
optimal parameters, which might prove difficult for traditional methods but ideal for GAs. However, because of its outstanding performance in optimization.

GAs were introduced as a computational analogy of adaptive systems. They are modeled loosely on the principles of the evolution via natural selection, employing a population of individuals that undergo selection in the presence of variationinducing operators such as mutation and recombination (crossover). A fitness function is used to evaluate individuals, and reproductive success varies with fitness.

The MSA Agent Algorithm: The proposed algorithm is working on the concept of genetic algorithm, which is capable to generate around 2600 results for various parameters and very much helpful to draft the various parameters for Rectangular and $U$ slit loaded micro strip antenna. The number of generations $(\mathrm{G})$ for the agent software can be find by the following formulation:

$$
\mathrm{G}=\mathrm{P}(\mathrm{n}) * \mathrm{X}(\mathrm{X}+22)
$$

Where $\mathrm{P}$ are the permutation of the total number of elements fed by user and generated by the agent . X are the variable parameters fed user.

The steps for the MSA Agent algorithm will be as follows

- $\quad$ Start and randomly generate an initial population $\mathrm{M}(\mathrm{x})$

- Compute and save the fitness for each individual $\mathrm{x}$ in the current population

- Define selection probabilities for each individual $n$ in $\mathrm{M}(\mathrm{t})$ (total population)
- Generate $\mathrm{M}(\mathrm{t}+1)$ by probabilistically selecting individuals from $\mathrm{M}(\mathrm{t})$ to produce offspring .

- $\quad$ Repeat step 2 until satisfying solutions are obtained.

The paradigm of GAs described above is usually the one applied to solving most of the problems presented to Rectangular and $\mathrm{U}$ slotted microstrip antenna. Though it might not find the best solution. more often than not, it would come up with a partially optimal solution.

The time complexity [9] For the two extreme cases of building block scaling, uniform

and exponential, genetic algorithms with perfect mixing have time complexities of $O(m)$ and $O(m 2)$ respectively.

Figure 1 shows the pictorial representation of the MSA agent algorithm. After the start point the algorithm takes the initial input parameters $\mathrm{h}$, fr, $\mathcal{\varepsilon} \mathrm{r}$, flow, fhigh, R. Where $\mathrm{h}$ is the height of the substrate material, fr is the resonating frequency, $\mathcal{\varepsilon} r$ is the permittivity of the substrate, $\mathrm{R}$ is the cable resistance which is 50 $\Omega$. flow, fhigh, are the upper and lower frequencies of $\mathrm{U}$ slit loaded microstrip antenna. The algorithm is divided into two sub parts, first part will calculate the various parameters for rectangular microstrip antenna and the second part will generate the population for $U$ slit loded microstrip antenna. And the fitness function will be applied to the parameters. Mutations will be performed after applying fitness function on both subparts. If there is variation in the parameters like $\mathrm{h}, \mathrm{fr}, \boldsymbol{\varepsilon} \mathrm{r}$, flow, fhigh, $\mathrm{R}$ is needed the pointer will jump back to step 3 and the process will be continued till the best optimized solutions are obtained.

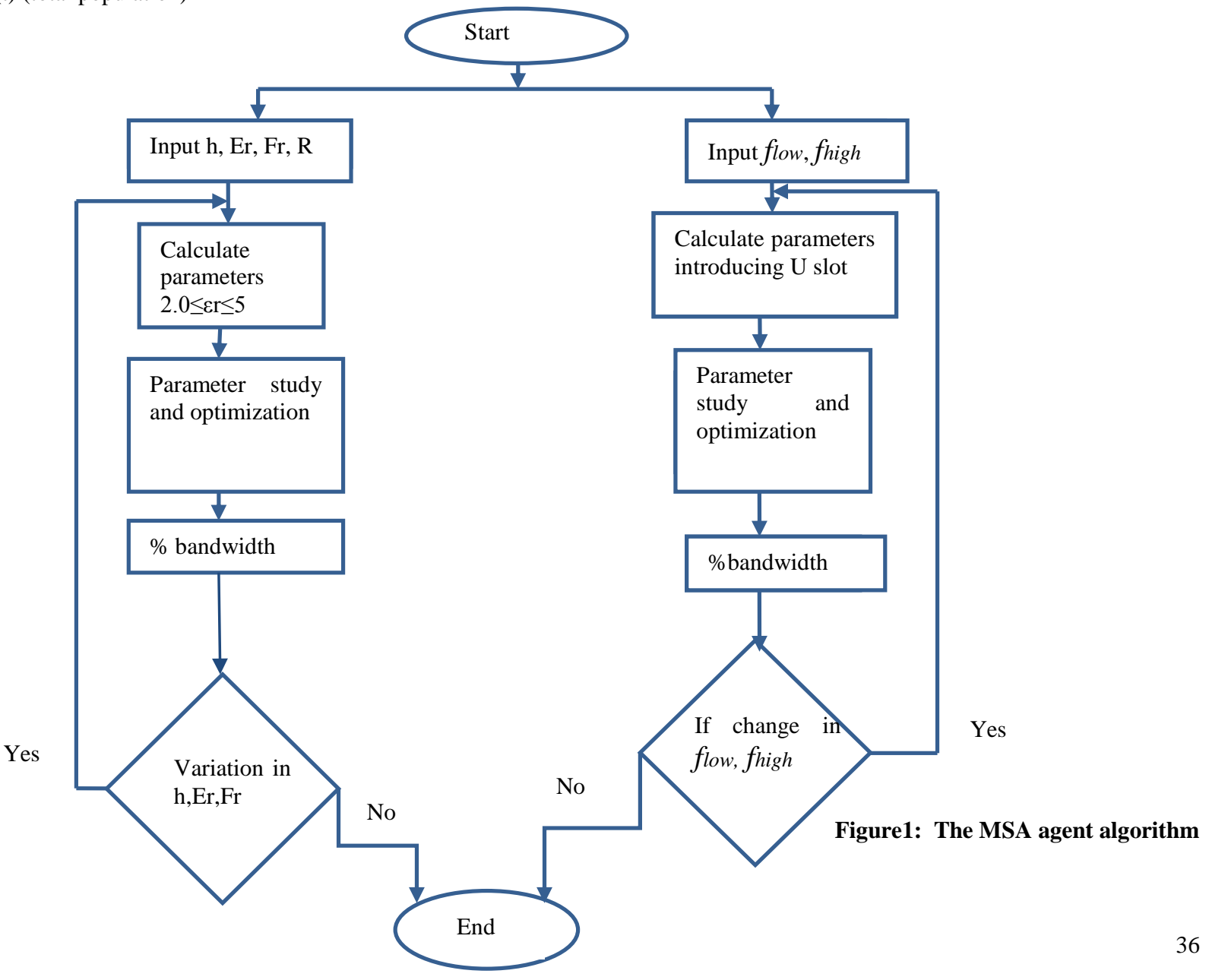




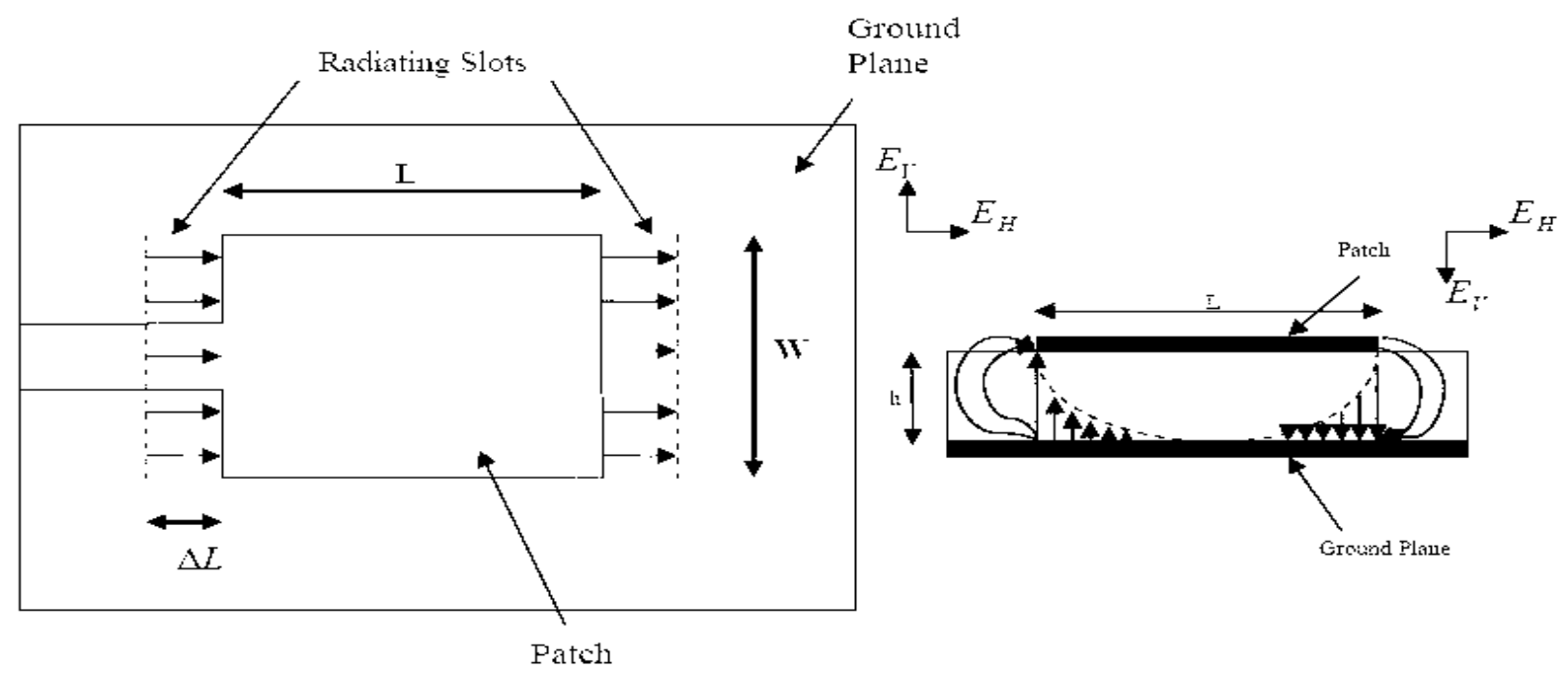

Figure 2: The Rectangular Microstrip antenna

\section{FORMULATION AND IMPLEMENTATION FOR THE OPTIMIZATION OF THE MICROSTRIP ANTENNA IN JAVA}

The configuration of the proposed patch antenna parasitic and driven is illustrated in Figure 2 and Figure 3 respectively. For the U-slotted patch, the slots are embedded to the rectangular patch. Where, $\mathrm{L}$ and $\mathrm{W}$ are the length and width of the patch. $\varepsilon \mathrm{r}$ is the dielectric constant, $\Delta \mathrm{L}$ is the length due to the fringing field.The fringing fields along the width can be model as radiating slots and electrically the patch of the micro strip antenna looks greater than its physical dimensions. The dimensions of the patch along its length have now been extended on each end by a distance $\Delta L$, which is given empirically by Hammers tad [1] as:

$$
\begin{gathered}
\Delta L=0.412 h((\text { creff }+0.3)(W / h+0.264) /(\text { creff } \\
-0.258)(W / h+0.8)
\end{gathered}
$$

The block of code for various formulas to design rectangular microstrip antenna in java is given as:

$$
\text { dl }=((0.412 * \mathrm{~h}) *(\mathrm{ee}+0.3) *((\mathrm{w} / \mathrm{h})+0.264)) /((\mathrm{ee}-
$$

$0.258) *((\mathrm{w} / \mathrm{h})+0.8))$

$\mathrm{W}=2 *$ Math.PI*fr;

$\operatorname{lmd}=\mathrm{c} / \mathrm{fr} ;$

$\mathrm{w}=(\mathrm{c} /(2 * \mathrm{fr})) *$ Math.sqrt $(2.0 /(\mathrm{er}+1))$

$\mathrm{ee}=((\mathrm{er}+1) / 2)+((\mathrm{er}-1) / 2) *$ Math.pow $((1+(12 * \mathrm{~h}) /(\mathrm{w})),(-0.5))$

$1=(\mathrm{c} /(2.0 * \mathrm{fr} *$ Math.sqrt $(\mathrm{ee})))-(2.0 * \mathrm{dl})$;

$\mathrm{G} 1=0.00836 *(\mathrm{w} / \mathrm{lmd})$;
$\operatorname{Rin}=1 /(2 * \mathrm{G} 1)$

y0 =(1/Math.PI $) *($ Math.acos $(($ Math.pow $($ Rf/Rin, 0.5$))))$;

$\mathrm{Bw}=3.77 *(((\mathrm{ee}-1) /($ Math.pow $(\mathrm{ee}, 2)) *(\mathrm{w} / \mathrm{l}) *(\mathrm{~h} / \mathrm{lmd})))$;

\section{U SLIT LOADED RECTANGULAR PATCH MICROSTRIP ANTENNA}

This design procedure is a set of simple design steps for the rectangular U-slot mircostrip patch antenna on microwave substrates. Determine centre frequency, fo Set center frequency as $f o$ and the lower and upper frequency bounds of the bandwidth as flow, fhigh, respectively.

Slot thickness $\mathrm{E}$ and $\mathrm{F}$ is defined as:

$$
\mathrm{E}=\mathrm{F}=\mathrm{lmd} / 60
$$

The implementation of various design formulas in java for $\mathrm{U}$ slit loaded microstrip antenna is as follows:

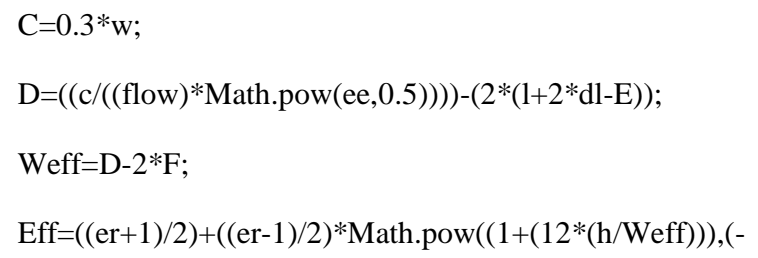


$\mathrm{H}=1-\mathrm{E}+2 * \mathrm{Dl}-((1 /($ Math.pow $(\mathrm{Eff}, 0.5))) *((\mathrm{c} /$ fhigh $)-(2 * \mathrm{C}+\mathrm{D}))$;

$\mathrm{G} 2=0.00836 *(\mathrm{w} / \mathrm{lmd})$;

$\operatorname{ppRin}=1 /(2 * \mathrm{G} 2)$;

ppy0 =(1/Math.PI $) *($ Math.acos $(($ Math.pow $($ Rf/ppRin, 0.5$)))) ;$

$\mathrm{BW}=3.77 *(($ Eff-1)/Math.pow $($ Eff, 2$)) *(\mathrm{w} / \mathrm{L}) *(\mathrm{~h} / \mathrm{lmd}))$;

\subsection{Validation}

Check that the sum $C+E+H$ is less than $L$. If not, need to adjust value of $C$ and $H$ until the design is physically possible.

Using the calculated values of $C, E$ and $H$, the total value exceeded the length of the antenna. Hence, the design is physically impossible. In order to make the design realizable, there is a need to change the initial lower, flow, and upper bound frequency, fhigh.

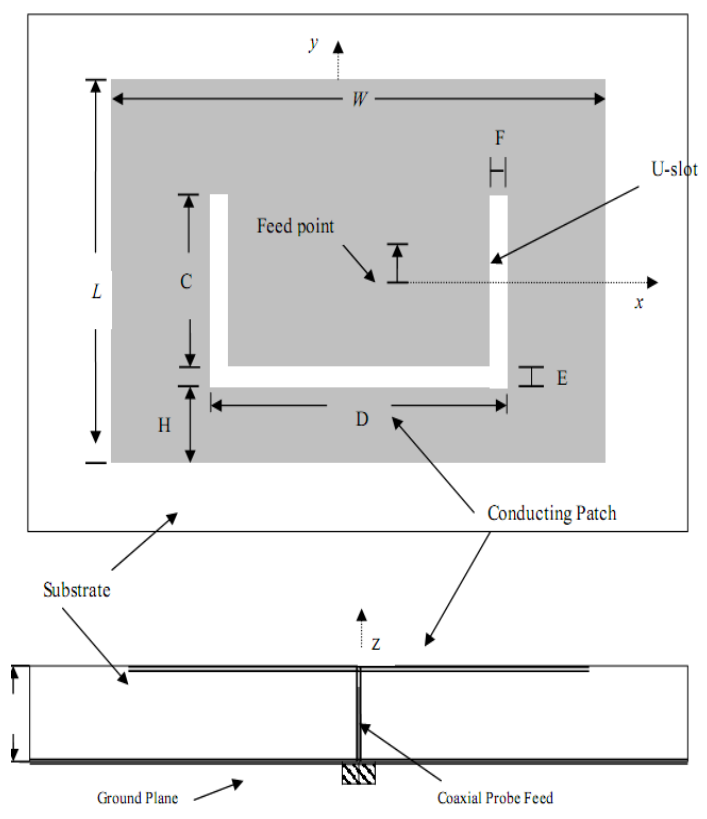

Figure 3: U slit loaded rectangular patch

\subsection{Verification and Experiment:}

The algorithm introduced in this paper was implemented in Java, We can browse the java program through Java Run Environment . The java applet which can run on any computer under the operating system of that computer, it is an application program that can react to user input and dynamically change. The reason that JRE is chosen that it can be executed anywhere, it can download java applet without viral infection. The reason behind choosing the java applet is to make our software a Graphical User Interface based, platform independent, The computation speed, precompiled code etc. Once the program is compiled, Various values can be tested for the analysis and results without compiling the program again and again. The program also shows a good agreement with Matlab simulated results. The implementation of the genetic algorithm gives a potential power to the software which can search approximately 2600 results.To test the microstrip antenna agent we used various simulated results available in research papers. The experiment indicates that the results by the program and the simulated results shows a very good agreement.

Using the optimization program, the user can set the lower bound and upper bound frequency to derive the bandwidth, while the dielectric constant is varied, Height $=10 \mathrm{~mm}$ of patch antenna. The optimized lower bound frequency, f low $=4 \mathrm{GHz}$, upper bound frequency, $\mathrm{f}$ high $=6 \mathrm{GHz}$, Resonant Frequency Fr=5 Ghz, wire resistance $=50 \mathrm{ohm}$ is selected , after going through various substrate values using the optimization program the design parameters has derived and the bandwidth has been optimized.

When $\varepsilon r=2.2$, Height $=10 \mathrm{~mm}$, lower bound frequency, $\mathrm{flow}=$ $4 \mathrm{GHz}$, upper bound frequency, $\mathrm{f}$ high $=6 \mathrm{GHz}$,Resonant Frequency $\mathrm{Fr}=5 \mathrm{Ghz}$ is selected for the parasitic patch and driven patch the computed results are shown in figure 4

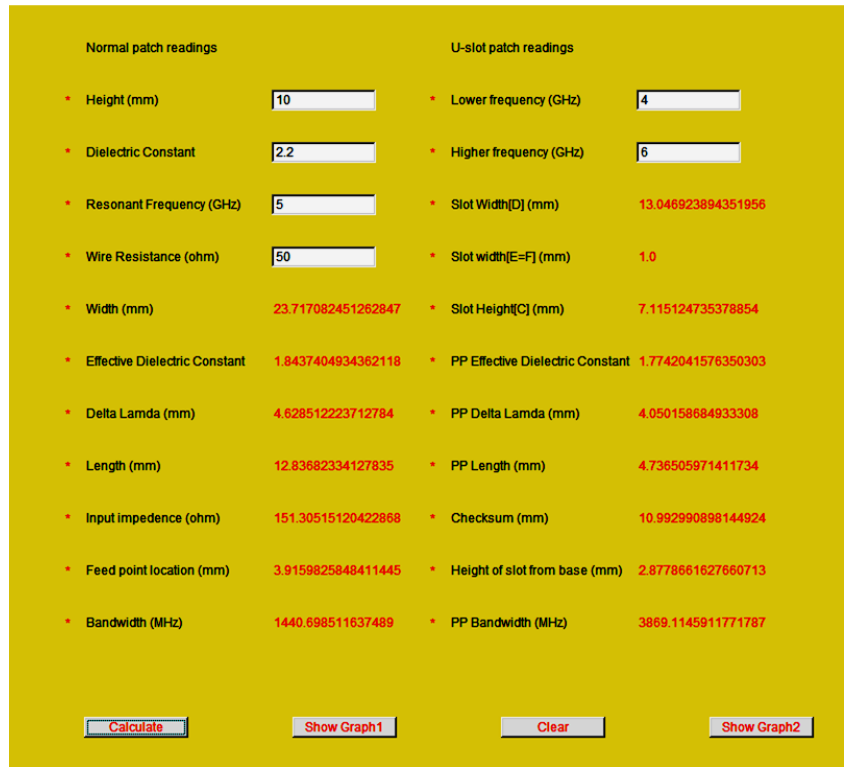

Figure 4: Interface when permittivity is $\mathbf{2 . 2}$

When $\varepsilon r=2.32$, Height $=10 \mathrm{~mm}$, lower bound frequency, $\mathrm{f}$ low $=4 \mathrm{GHz}$, upper bound frequency, $\mathrm{f}$ high $=6 \mathrm{GHz}$, Resonant Frequency $\mathrm{Fr}=5 \mathrm{Ghz}$ is selected for the rectangular and $\mathrm{U}$ slot patch the computed results are shown in figure 5 


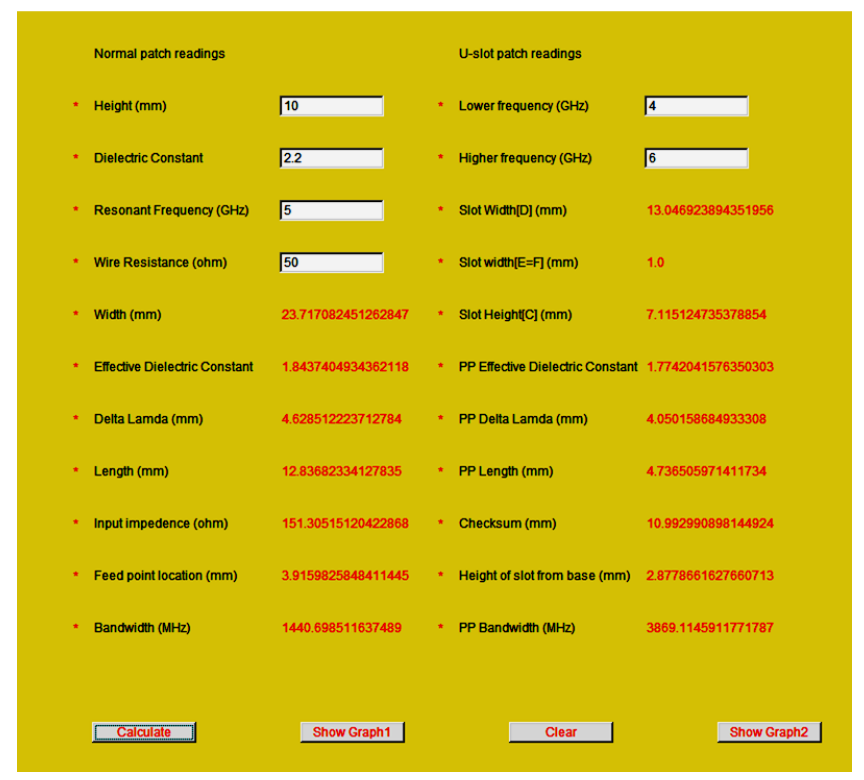

Figure 5: Interface when permittivity is $\mathbf{2 . 3 2}$

When $\varepsilon r=2.6$, Height $=10 \mathrm{~mm}$, lower bound frequency, $\mathrm{flow}=$ $4 \mathrm{GHz}$, upper bound frequency, $\mathrm{f}$ high $=6 \mathrm{GHz}$, Resonant Frequency $\mathrm{Fr}=5 \mathrm{Ghz}$ is selected for the rectangular and $\mathrm{U}$ slot patch the computed results are shown in figure 6

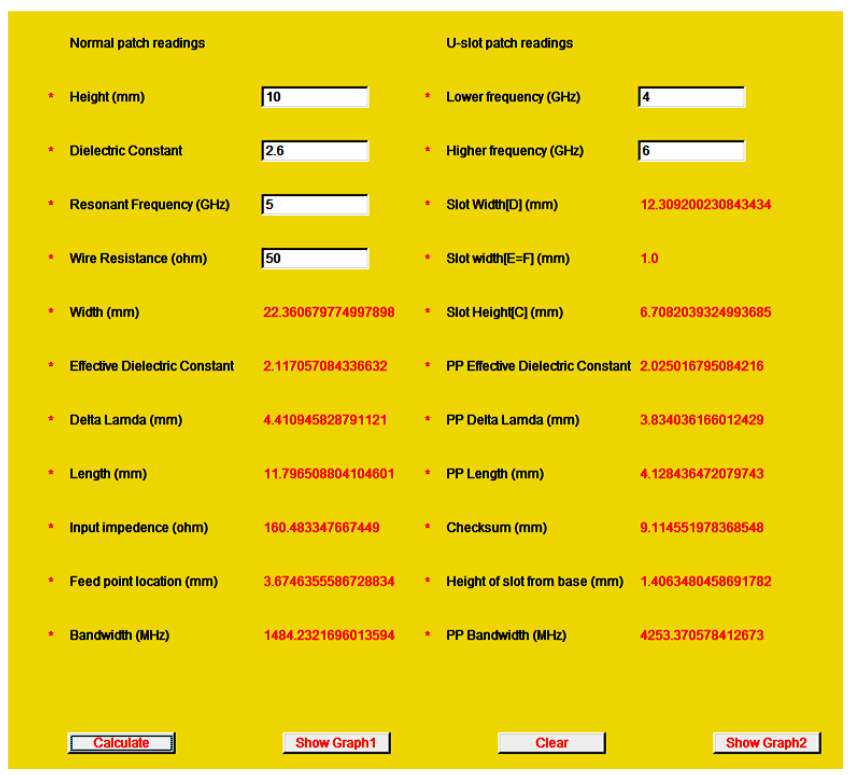

Figure 6: Interface when permittivity is $\mathbf{2 . 6}$

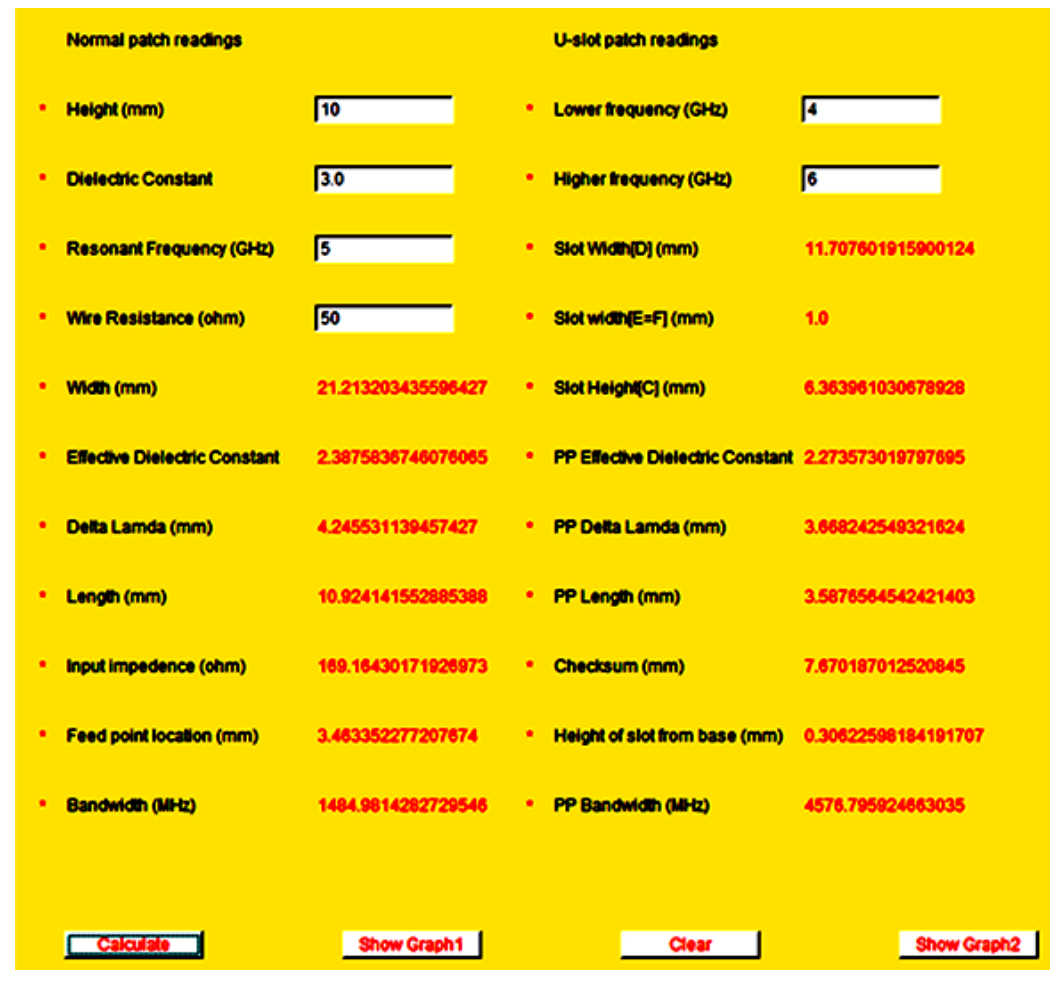

Figure 7: Interface when permittivity is 3.0

When $\varepsilon r=3.0$, Height $=10 \mathrm{~mm}$, lower bound frequency, $\mathrm{flow}=$ $4 \mathrm{GHz}$, upper bound frequency, $\mathrm{f}$ high $=6 \mathrm{GHz}$, Resonant Frequency $\mathrm{Fr}=5 \mathrm{Ghz}$ is selected for the rectangular and $\mathrm{U}$ slot patch the computed results are shown in figure 7 


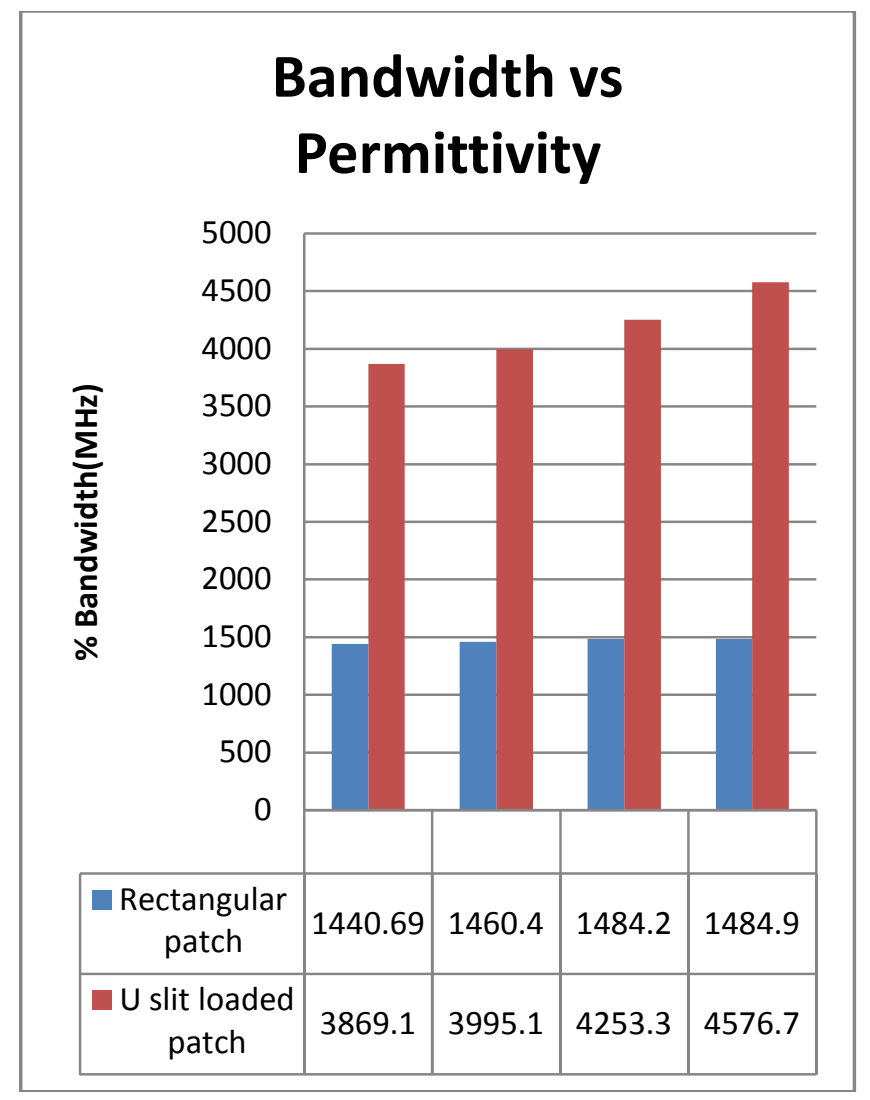

Figure 8:The bandwidth vs permittivity

\section{CONCLUSION}

Hence, it is proven by the results, by changing the permittivity and introducing the slot (U) to the rectangular microstrip antenna, for the resonant frequency $5 \mathrm{GHz}$ at the frequency range $4 \mathrm{GHz}$ to $6 \mathrm{GHz}$, and introducing the slot $\mathrm{U}$ the optimized results are obtained. The results are simulated with pre research done .The result shows a very good agreement between simulation results and the MSA agent result.

\section{REFERENCES}

[1] G.A Deschamps, "Microstrip Microwave Antennas" Presented at the third USAF symp. On antenna, 1953.

[2] Rashid A. Bhatti, Nguyen Viet Anh, and Seong-Ook Park," Pattern Recon ${ }^{-}$gurable Antenna Array for MIMO-enabled Handheld Wireless Communication Devices “,Progress In Electromagnetics Research Symposium Abstracts, Beijing, China, March 23\{27, 2009

[3] Jia-Yi Sze; Kin-Lu Wong," Slotted rectangular microstrip antenna for bandwidth enhancementon ",IEEE Transactions Antenna and propagation, Aug 2000

[4] H.Gutton and G.Baissinot, "Flat Aerial for High Frequencies" French patent No.703113, 1955.

[5] Neela Chattoraj and Jubendu Sekhar Roy "Application of genetic algorithm to the optimization of gain of magnetized ferrite microstrip antenna" , Engineering Letters 14:2,EL_14_2_15,16 May 2007

[6] R. Garg, P. Bhartia, I. Bahl and A. Ittipiboon, "Microstrip Antenna Design Handbook", Artech House Antennas \& Propagation Library, (Nov 2000)

[7] A.K. Gautam and B. R. Vishwakarma, "Frequency agile Micro strip antenna using symmetrically loaded tunnel Diode "Indian radio and space physics, June 2006

[8] Vinod K. Singh, Zakir Ali,” Dual Band U- shaped microstrip antenna for wireless Communication", International Journal of Engineering Science and Technology Vol. 2(6), 2010, 1623-1628

[9] Fernando G. Lobo, David E. Goldberg and Martin Pelikan,' Time complexity of genetic algorithms on exponentially scaled problems", June 2005 